\section{Temperature responsive phosphorescent small unilamellar vesicles $\nmid$}

\author{
Mouchumi Bhuyan and Burkhard Koenig* \\ Received 7th May 2012, Accepted 7th June 2012 \\ DOI: $10.1039 / \mathrm{c} 2 \operatorname{cc} 33279 \mathrm{e}$
}

Self-assembled lipid vesicles with embedded amphiphilic terbium(III) complexes show a strong temperature dependence of their phosphorescence intensity and lifetime in the physiological range.

Temperature is a fundamental physical property of matter important in everyday life, in scientific and industrial applications. The methods to measure temperature can be divided into two main techniques: contact thermometry and non-contact thermometry. Contact methods use thermocouples, thermistors, and resistance temperature detectors (RTDs), whereas noncontact methods often use the spectral emittance of a material for readout. ${ }^{1}$

Non-contact optical techniques have various advantages in terms of sensitivity and real-time monitoring over a wide range, from femtoseconds to seconds. ${ }^{2}$ Among the available optical methods, infrared thermometry that uses the principle of blackbody radiation is flexible and easy to use, but can only measure the temperature of surfaces, thus limiting its applications. ${ }^{3}$ Therefore luminescence based optical sensors have attracted much attention, because of their fast response, high spatial resolution, accurate usability even in strong electromagnetic fields ${ }^{4}$ and safety of remote handling. ${ }^{5}$ Such sensing probes use appropriate luminophores ranging from polycyclic aromatic hydrocarbons to metal complexes. ${ }^{2}$ Temperature sensitive probes were incorporated into nanogels for intracellular thermometry, ${ }^{6}$ and in sensor films or thermo-sensitive polymers to allow spatially resolved temperature imaging. ${ }^{7}$

Recently tripositive lanthanide complexes have been used for optical sensing of temperature. ${ }^{4,7,8}$ The use of such lanthanide complexes has several advantages over organic luminophores: (a) they have long emission wavelengths, (b) lanthanide emissions are Laporte forbidden and are therefore characterized by very long luminescence lifetimes, which is useful for applications in biological media by excluding background emission using time delayed detection, (c) 4 f electrons of the lanthanides are highly shielded from the chemical environment by the $5 \mathrm{~s}$ and $5 \mathrm{p}$ orbitals thus making them insensitive to the environment. Among the tripositive lanthanides, typically $\mathrm{Eu}^{3+}$ based complexes have been used as optical temperature sensors,

Institut für Organische Chemie, Universität Regensburg, Regensburg, 93040, Germany. E-mail: burkhard.koenig@chemie.uni-regensburg.de; Fax: +49-941-943-1717; Tel: + 49-941-943-4575

$\dagger$ Electronic supplementary information (ESI) available: Experimental details, characterisation of synthesized complexes and vesicles, temperature dependent lifetime and emission intensity data of the vesicles. See DOI: 10.1039/c2cc33279e
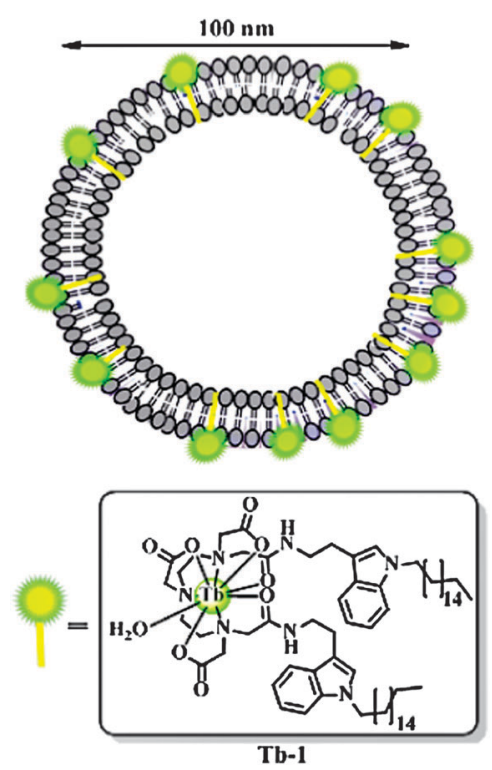

Fig. 1 Schematic representation of LNT.

while $\mathrm{Tb}^{3+}$ complexes are less explored. One reason is their smaller luminescence dependence on temperature. ${ }^{9}$ However, the longer luminescence lifetime of the $\mathrm{Tb}^{3+}$ ion compared to $\mathrm{Eu}^{3+}$ makes $\mathrm{Tb}^{3+}$ complexes the favoured systems for the lifetime based optical sensing of temperature. ${ }^{10}$ Having this in mind we used the diethylenetriamine pentaacetic acid (DTPA) based amphiphilic $\mathrm{Tb}^{3+}$ complex (Tb-1) as a probe for the optical sensing of temperature. By embedding the complex [for detailed experimental procedures and analytical data of the prepared compounds, see ESI $\dagger$ ] in different phospholipids we obtain reversible thermosensitive luminescent vesicles (Fig. 1) at an adjustable temperature range. The luminescent nanosized vesicles (LNT) with embedded $\mathrm{Tb}^{3+}$ complexes were prepared by the previously reported film-hydration method. ${ }^{11}$ The multilamellar vesicles were extruded through a membrane yielding unilamellar vesicles of $90-100( \pm 5) \mathrm{nm}$ in size (see Fig. S11 and S12 in ESI $\dagger$ ). We have used different commercially available phospholipids for the preparation of LNT 1-6 (Table 1).

The emission intensities of lanthanide complexes depend on their concentration. ${ }^{13}$ Therefore several different concentrations of Tb-1 were used in the lipid membranes and in terms of emission intensity, $5 \mathrm{~mol} \%$ of $\mathbf{T b}-\mathbf{1}$ with respect to the used lipids is the most suitable condition for these systems. 
Table 1 Composition response range of LNT 1-6

\begin{tabular}{|c|c|c|c|c|}
\hline Vesicles & $\begin{array}{l}\text { Phospholipids } \\
\text { used }\left[T_{\mathrm{m}}{ }^{a}\left({ }^{\circ} \mathrm{C}\right)\right]\end{array}$ & $\begin{array}{l}\text { Effective } \\
\text { range }\left({ }^{\circ} \mathrm{C}\right)\end{array}$ & $\begin{array}{l}\text { Most } \\
\text { sensitive } \\
\text { range }^{b}\left({ }^{\circ} \mathrm{C}\right)\end{array}$ & $\begin{array}{l}\text { Sensitivity }{ }^{b} \\
\left(\%{ }^{\circ} \mathrm{C}^{-1}\right)\end{array}$ \\
\hline LNT1 & $18: 0$ PC (DSPC) [55] & $0-60$ & $25-55$ & \\
\hline LNT2 & $16: 0 \mathrm{PC}$ (DPPC) & & & \\
\hline LNT3 & $14: 0$ PC (DMPC) [23] & $0-25$ & 10 & -4 . \\
\hline LNT4 & DSPE-PEG350 [ 60] & $0-60$ & $5-5$ & -1.8 \\
\hline LNT5 & $\begin{array}{l}18: 1 \mathrm{c} 9 \text { PC (DOPC) } \\
{[-20]}\end{array}$ & n.a. & n.a. & n.a. \\
\hline LNT6 & $\begin{array}{l}\mathrm{PC}(18: 0 / 14: 0) \\
(\mathrm{SMPC})[30]\end{array}$ & $0-30$ & $15-30$ & -2.8 \\
\hline $\begin{array}{l}\text { LNT4 }^{c}+ \\
\text { BSA }\end{array}$ & DSPE-PEG350 [ 60] & $0-60$ & $25-55$ & -1.4 \\
\hline \multicolumn{5}{|c|}{$\begin{array}{l}{ }^{a} T_{\mathrm{m}}=\text { phase transition temperature of lipid. }{ }^{b} \text { The most sensitive } \\
\text { temperature range and sensitivity per }{ }^{\circ} \mathrm{C} \text { are based on the temperature } \\
\text { dependent lifetime measurements carried out in HEPES buffer at } \mathrm{pH} 7.4 \text {. } \\
{ }^{c} \text { Non-specific interactions between the constituent lipids and BSA }{ }^{12} \text { are } \\
\text { minimised by shielding the vesicular surface using DSPC-PEG350 having } \\
\text { oligoethylene glycol residues attached to its polar head groups. }\end{array}$} \\
\hline
\end{tabular}

All vesicles (LNT) containing the $\mathrm{Tb}^{3+}$ complex exhibit a phosphorescence emission intensity at 488, 545, 584, $620 \mathrm{~nm}$, corresponding to the electronic transition from the ${ }^{5} \mathrm{D}_{4}$ state to ${ }^{7} \mathrm{~F}_{3},{ }^{7} \mathrm{~F}_{4},{ }^{7} \mathrm{~F}_{5},{ }^{7} \mathrm{~F}_{6}$ states of the $\mathrm{Tb}^{3+}$ ions. However, at $25{ }^{\circ} \mathrm{C}$, the emission intensity is significantly different for LNT1 (DSPC lipids, $T_{\mathrm{m}}=55^{\circ} \mathrm{C}$ ) and LNT5 (DOPC lipids, $T_{\mathrm{m}}=-20{ }^{\circ} \mathrm{C}$ ) (see Fig. S1 in ESI $\dagger$ ) which indicates that the phase transition temperature of the lipids affects the emission intensity.

The temperature dependent emission intensity and lifetime measurements of the vesicular systems LNT were carried out mainly in HEPES buffer at $\mathrm{pH}$ 7.4. There is no significant effect on the emission temperature response by $\mathrm{pH}$ changes in the range from $\mathrm{pH} 6$ to 8 , by changing the buffer substance or varying the salt concentration (for details see ESI $\dagger$, Fig. S7 and S8). All vesicles show a decrease in emission intensity with
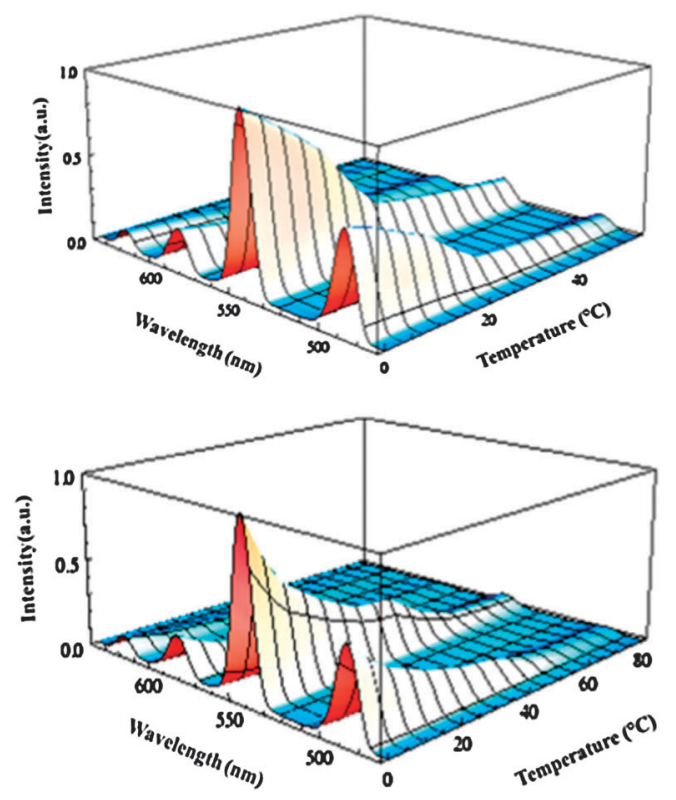

Fig. 2 Temperature dependence of emission intensity measurements for LNT3 [top] and LNT4 [bottom] [ $\lambda_{\mathrm{ex}}=285 \mathrm{~nm}$; conc. of Tb(III) $=$ $\left[5 \times 10^{-6} \mathrm{M}\right]$.

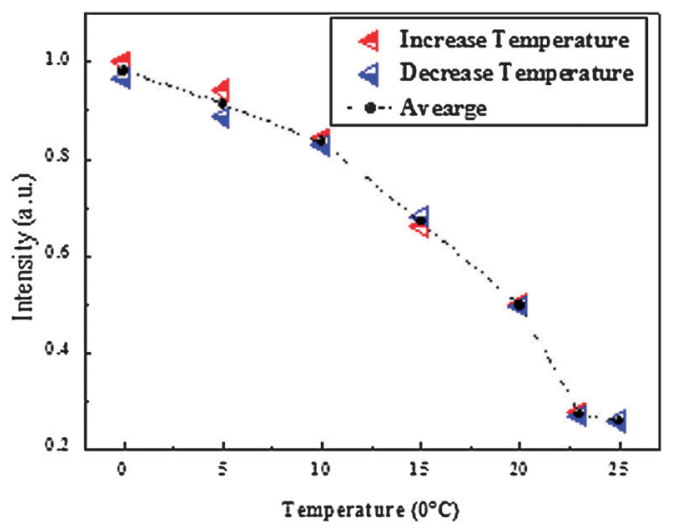

Fig. 3 Reversibility of luminescence intensity of LNT3 in the measured temperature range (changes at $545 \mathrm{~nm}$ emission wavelength are shown).

an increase in temperature and each system shows more than $85 \%$ decrease in luminescence intensity around the phase transition temperature of the constituent lipid used (Fig. 2).

The emission changes for the vesicular systems, LNT 1-6 (except LNT5), are reversible within the measured temperature range, as exemplarily shown in Fig. 3 for LNT3.

However the emission intensity based measurements often suffer from changing sensor concentrations or drifts of the optoelectronic systems (lamps and detectors), ${ }^{8 c}$ whereas decay lifetime based data are free from these drawbacks and are more reliable. ${ }^{8 c}$ The synthesised luminescent vesicular systems, LNTs, also show reversible changes in the decay lifetimes with varying temperature. The luminescence decay vs. time curve follows a first order exponential decay at every measured temperature (see Fig. S4 in ESI $\dagger$ ) and the average lifetime $(\tau)$ is determined by eqn $(1)$,

$$
y=A_{1} * \exp (-x / \tau)+y_{0}
$$

where $y=$ lifetime at time $x ; y_{0}=$ initial lifetime; $x=$ time; $\tau=1 /$ (decay constant); $A_{1}=$ pre-exponential factor.

The lifetime $v s$. temperature plot shows that for every LNT, average lifetime $(\tau)$ decreases with an increase in temperature and the system shows high sensitivity around the phase transition temperature $\left(T_{\mathrm{m}}\right)$ of the constituent lipid (Fig. 4). Thus the most sensitive temperature range for these nanosystems can be tuned by changing the constituent lipids (Table 1).

The luminescence intensity and lifetime of lanthanides can be affected by many factors. The overall decay rate constant of the luminescence level can be expressed by the following equation, ${ }^{14}$ where $k_{\mathrm{r}}$ is the radiative rate constant and $k_{\mathrm{nr}}$ and $k_{\mathrm{nr}}(T)$ are the non-radiative temperature independent and temperature dependent decay rate constants. ${ }^{14}$

$$
k=\frac{1}{\tau}=k_{\mathrm{r}}+k_{\mathrm{nr}}+k_{\mathrm{nr}}(T)
$$

The non-radiative transition consists of the vibrational excitation, back energy transfer (BET) to the triplet excited state of the attached sensitizer from the ${ }^{5} \mathrm{D}_{4}$ state of $\mathrm{Tb}(\mathrm{III})$ and the concentration quenching. The temperature-dependence of the decay rate constant is expected for the vibrational excitation and $\mathrm{BET}$ in the non-radiative transition from the ${ }^{5} \mathrm{D}_{4}$ level of the terbium(III) ion. ${ }^{9 a}$ A decrease in luminescence intensity can therefore be related to both of these factors, while the decrease 


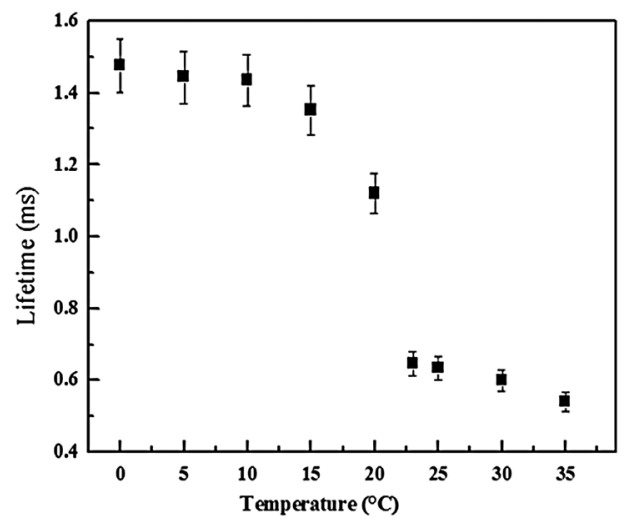

Fig. 4 Average lifetime $\left( \pm\right.$ s.d) (ms) vs. temperature $\left({ }^{\circ} \mathrm{C}\right)$ plot for LNT3

in luminescence lifetime depends only on the energy dissipation from the ${ }^{5} \mathrm{D}_{4}$ state of $\mathrm{Tb}(\mathrm{III})$. These facts give a rationale for the sensitivity of LNT near the transition temperature of the lipid. The constituent phospholipids are in a tightly packed gel phase below the transition temperature $\left(T_{\mathrm{m}}\right)^{15}$ leading to deactivation of the vibrational excitation and hence a longer lifetime of the embedded Tb(III) ion. Near or above the phase transition temperature, the lipid bilayers are more or less in the liquid crystalline phase $\mathrm{e}^{15 c}$ with higher chain mobility of the constituent lipids resulting in a drastic decrease in the luminescence lifetime by vibrational dissipation of energy from the ${ }^{5} \mathrm{D}_{4}$ state of the $\mathrm{Tb}(\mathrm{III})$ ion. Above $T_{\mathrm{m}}$ of the corresponding lipids, the nanosystems show negligible sensitivity towards an increase in temperature. The phase transition temperature is not affected by $\mathrm{pH}$ changes in the range from $\mathrm{pH} 6$ to 8 allowing the application of the system in this range.

The fully PEGylated vesicle, LNT4, shows reversible change in emission intensity with temperature. However, for a given wavelength, the intensity vs. temperature plot is convex for the non-PEGylated vesicles, while it is concave for the completely PEGylated vesicular system, LNT4 (Fig. 2 (bottom). The difference in the nature of the plot can be attributed to increased chain mobility of the PEGylated lipids as shown by Belsito et al. using spin-label ESR measurements. ${ }^{16}$ The temperature dependent luminescence changes of LNT4 are not affected by the presence of bovine serum albumin (BSA, Table 1). The Tb-functionalized vesicles are effective in regular cell culture medium (DMEM, 10\% FCS), however, the presence of phenol red in standard solutions interferes with the absorption of the dyes and thereby lowers the emission intensity.

In summary, we have developed a nanosized luminescent thermometer by self-assembly of lipids and amphiphilic Tb(III) complexes. These aggregates find applications in sensing and imaging of physiological temperatures by observing changes of their phosphorescence intensity and lifetime. The most sensitive temperature and sensitivity per ${ }^{\circ} \mathrm{C}$ can be tuned by merely varying the phase transition temperature of the constituent lipids as demonstrated by several examples. The membranes can be processed, e.g. by simple spreading on a glass surface without losing their sensing ability. Since Tb(III) has a very long luminescence lifetime, possible interference of background emission arising from proteins or cells can be avoided by delayed readout.

MB thanks the Bavarian Research foundation for graduate fellowship. We thank the University of Regensburg and the Deutsche Forschungsgemeinschaft for support of our research.

\section{Notes and references}

1 (a) R. E. Bentley, Temperature and Humidity Measurement, Springer, Singapore, 1998; (b) http://www.temperatures.com.

2 M. Mitsuishi, S. Kikuchi, T. Miyashita and Y. Amao, J. Mater. Chem., 2003, 13, 2875.

3 E. F. J. Ring, Infrared Phys. Technol., 2007, 49, 297.

4 S. M. Borisov and O. S. Wolfbeis, Anal. Chem., 2006, 78, 5094.

5 R. Schorer, E. Friess, K. Eberl and G. Abstreiter, Phys. Rev. B, 1991, 44, 1772.

6 S. Uchiyama, A. Prasanna de Silva and K. Iwai, J. Chem. Educ., 2006, 83, 720 .

7 G. E. Khalil, K. Lau, G. D. Phelan, B. Carlson, M. Gouterman, J. B. Callis and L. R. Dalton, Rev. Sci. Instrum., 2004, 75, 192.

8 (a) H. Lam, G. Rao, J. Loureiro and L. Tolosa, Talanta, 2011, 84, 65; (b) Y. Ohishi and S. Takahashi, Appl. Opt., 1986, 25, 720; (c) H. Peng, M. I. J. Stich, J. Yu, L. Sun, L. H. Fischer and O. S. Wolfbeis, Adv. Mater., 2010, 22, 716; (d) Y. Dwivedi and S. B. Rai, Sens. Actuators, A, 2010, 163, 37; (e) H. Sakaue, C.-Y. Huang and J. P. Sullivan, Sens. Actuators, B, 2011, 155, 372

9 (a) S. Katagiri, Y. Tsukahara, Y. Hasegawa and Y. Wada, Bull. Chem. Soc. Jpn., 2007, 80, 1492; (b) L.-N. Sun, J. Yu, H. Peng, J. Z. Zhang, L.-Y. Shi and O. S. Wolfbeis, J. Phys. Chem. C, 2010, 114, 12642.

10 (a) M. D. Chambers and D. R. Clarke, Surf. Coat. Technol., 2007, 202, 688; (b) D. R. Clarke and M. M. Gentleman, Surf. Coat. Technol., 2007, 202, 681.

$11 \mathrm{~J}$. Lasch, V. Weissig and M. Brandl, in Liposomes, ed. V. Torchilin and V. Weissig, Oxford University Press, 2nd edn, 2003.

12 O. D. Velev, Adv. Biophys., 1997, 34, 139.

13 (a) D. de Graaf, S. J. Stelwagen, H. T. Hintzen and G. de With, J. Non-Cryst. Solids, 2003, 325, 29; (b) J. Hölsä, M. Leskelä and L. Niinistö, Mater. Res. Bull., 1979, 14, 1403.

14 N. Sabbatini, M. Guardigli and J.-M. Lehn, Coord. Chem. Rev., 1993, 123, 201.

15 (a) J. H. Crowe, A. E. Oliver, F. A. Hoekstra and L. M. Crowe, Cryobiology, 1997, 35, 20; (b) W. F. Wolkers, H. Oldenhof and B. Glasmacher, Cryobiology, 2010, 61, 108; (c) M. Caffrey and J. Hogan, Chem. Phys. Lipids, 1992, 61, 1.

16 S. Belsito, R. Bartucci, G. Montesano, D. Marsh and L. Sportelli, Biophys. J., 2000, 78, 1420. 